\title{
Épurer et punir : la justice confrontée à ses dilemmes
}

\author{
Danièle Lochak
}

A propos de...

— JeAn Jean-Paul (dir.), Juger sous Vichy, juger Vichy, Paris : La Documentation française, 2018, $451 \mathrm{p}$.

— MouRALIS Guillaume, Le moment Nuremberg : le procès international, les lawyers et la question raciale, Paris : Sciences Po Les Presses, 2019, 255 p.

Quel sort réserver aux auteurs des crimes commis pendant la Seconde Guerre mondiale? Au-delà de ce questionnement commun, les deux ouvrages analysés sont, par leur contenu et leur problématique, assez différents l'un de l'autre.

Le premier, publié dans la collection «Histoire de la Justice» éditée par la Documentation française, rassemble des contributions parues dans des livraisons précédentes de la collection. Elles traitent à la fois, comme le nom de l'ouvrage l'indique, de la justice sous Vichy: du fonctionnement des juridictions d'exception, de l'attitude des magistrats et des avocats face à la collaboration et à la Résistance - et de la place et du rôle dévolus à la justice dans le processus d'épuration à la Libération.

L'ouvrage de Guillaume Mouralis, délaissant le déroulement proprement dit du procès de Nuremberg et la sentence qui en a été l'aboutissement, se propose d'analyser, en amont, ses conditions de possibilité ou encore, pour reprendre la terminologie de l'auteur, " les coulisses et la machinerie » du tribunal et, en aval, le « legs » de Nuremberg. Il montre comment l'idée en a germé puis s'est concrétisée, il décrit les divergences et la concurrence entre groupes de juristes d'origines et de cultures différentes, dotés de ressources académiques et politiques inégales, les rapports de force entre universitaires - globalement tenus à l'écart - et praticiens. Mais il croise avec cette problématique la "question raciale », déclinée sous deux aspects : comment juger les atrocités nazies inspirées par un racisme exacerbé, alors que le racisme est encore institutionnalisé aux États-Unis et profondément ancré dans les mentalités; symétriquement, jusqu'à quel point les acquis de Nuremberg ont-ils été mobilisés ultérieurement à l'appui du combat pour les droits civiques.

La lecture conjointe des deux ouvrages suppose de laisser de côté, sans pour autant minorer leur intérêt, certains éléments de leurs contenus respectifs 1 pour se concentrer sur les questions transversales qui s'en dégagent. Inversement, il a paru pertinent, pour éclairer ces questionnements, d'introduire des références à d'autres ouvrages ou contributions traitant des procès de dénazification en Allemagne après 1945 ou même d'un autre cas emblématique d'épuration : celle qui a visé les dirigeants et agents de la RDA après la réunification 2.

Certaines questions, qui surgissent de façon récurrente, nous serviront de fil conducteur. Pourquoi juger : quelle est la finalité de l'épuration et comment s'impose le choix de la voie judiciaire (I) ? Quelles sont les contraintes, de nature politique ou juridique, potentiellement contradictoires, qui vont conditionner certains choix (II) ? Quel droit appliquer, alors que les infractions reprochées n'étaient inscrites dans aucun texte au moment des faits - et faut-il se

1. Il s'agit notamment des contributions consacrées à " Juger sous Vichy », concernant le premier ouvrage et, pour le second, de l'analyse fine des trajectoires des juristes, avant, pendant et après le « moment Nuremberg ».

2. On fait ici allusion à l'ouvrage de Guillaume Mouralis tiré de sa thèse : Une épuration allemande. La RDA en procès (1949-2004), Paris : Fayard, 2008. 
référer au droit naturel quand le droit positif paraît inadéquat (III) ? Qui est compétent pour juger, question qui inclut celle de la légitimité de ceux qui vont juger (IV) ? Comment apprécier les responsabilités individuelles pour des crimes collectifs dont les auteurs pouvaient prétendre n'avoir été que les rouages d'un système qui les dépassait (V) ? Et finalement, quelles leçons ont été tirées de ces différentes expériences (VI)?

\section{Juger et punir : pourquoi, comment ?}

La question du jugement des criminels de guerre était loin d'être consensuelle au départ chez les Alliés. À l'issue de la conférence interalliée tenue à Londres en janvier 1942, les représentants des neuf gouvernements en exil avaient adopté une déclaration dans laquelle ils disaient «[placer] parmi les buts principaux de la guerre le châtiment, par les voies d'une justice organisée, des coupables ou responsables de ces crimes - qu'ils les aient ordonnés, perpétrés, ou qu'ils y aient participé ». Ceci n'empêche pas que se manifestent par la suite des divergences entre les pays alliés sur cette question du jugement des criminels de guerre, sur la forme et l'ampleur que devaient prendre les futurs procès - et même sur l'opportunité de la voie judiciaire. Churchill lui-même aurait déclaré à ses ministres, en décembre 1942: «If Hitler falls into our hands, we shall certainly put him to death ", émettant de fortes objections à l'égard de ce qui risquait de n'être qu'une parodie de procès compte tenu des problèmes juridiques délicats qu'il poserait au regard du droit international.

Au sein de l'administration Roosevelt, en septembre 1944, le secrétaire du Trésor, Henry Morgenthau, plaidait en faveur d'une paix «carthaginoise » incluant des exécutions sommaires de dirigeants nazis. Mais son collègue du département de la Guerre, Henry Stimson, s'opposa à ce plan et défendit auprès de Franklin Roosevelt l'idée d'un procès des « grands criminels de guerre » conforme aux principes juridiques libéraux. C'est cette idée qui l'emporta et un projet fut élaboré par les juristes du département, consigné dans un mémorandum: "Trial of European War Criminals », en septembre 1944 3. À partir du printemps 1945, il fit l'objet d'une négociation diplomatique avec les autres puissances alliées, pour aboutir à la signature de l'accord de Londres du 8 août 1945 et de son annexe portant statut du tribunal militaire international.

Dans le cas de Vichy, la question de l'épuration a émergé très tôt au sein du Comité français de libération nationale (CFLN) et dans les rangs de la Résistance. La Résistance intérieure réclamait une justice implacable, à l'image de ce tract parachuté en France en 1943 représentant une potence avec la légende suivante : "Où qu'ils soient, quoi qu'ils fassent les traîtres seront châtiés 4. » De Gaulle, lui, souhaitait limiter l'épuration aux personnages qui avaient joué un rôle éminent dans la politique de Vichy ou qui s'étaient faits les complices directs de l'ennemi. Désigner quelques coupables: « une poignée de misérables et d'indignes », selon la formule du Général de Gaulle, sur lesquels serait reportée toute la honte de la collaboration était une façon de consacrer le mythe d'une France globalement résistante qui n'avait pas accepté Vichy et l'occupation 5.

La question de l'épuration de la magistrature, en particulier, divisait les juristes 6. Les uns préconisaient la modération et la mise à l'écart des seules personnes qui, par leur comportement, s'étaient rendues indignes de leur fonction. Mais d'autres, au premier rang desquels les avocats communistes dont l'un des représentants assurait l'intérim du ministère

3. Guillaume MouraLis, Le moment Nuremberg, p. 49-50.

4. Jean-Louis CREMIEUX-BrilhaC, « Avant la Libération : la politique de la Justice à Alger et le procès Pucheu (1943-1944) », in Juger sous Vichy, juger Vichy.

5. Alain BANCAUD, « L'épuration judiciaire à la Libération : entre légalité et exception », in ibid.

6. Liora ISRAËL, « Les milieux judiciaires dans la Résistance et à la Libération », in ibid. 
de la Justice, estimaient qu'il fallait châtier ceux qui portaient une lourde part de responsabilité dans les souffrances et les deuils des Français et qu'il était juste qu'ils pâtissent dans leur carrière de leur défaut de courage civique et professionnel.

Mais tandis qu'on discute de la portée de l'épuration à venir, l'année 1944 est marquée par une phase d'épuration sauvage et sanglante: lynchages, exécutions sommaires, femmes tondues, jugement par des cours martiales improvisées composées de FTP et de FFI 7. Commencée sous Vichy, elle s'exacerbe pendant la période insurrectionnelle et se prolonge jusqu'à la fin 1945 sous des formes plus atténuées 8. Aux yeux du Général de Gaulle, l'essentiel est d'imposer l'autorité de l'État et de sa justice pour ne pas laisser se développer une épuration incontrôlée. Le recours au droit et à la justice est donc une façon de canaliser l'excès de violence, ce qui se traduira par une codification précise et détaillée de l'épuration 9 .

\section{Logiques politiques et contraintes juridiques}

Même lorsque le choix est fait d'une épuration judiciaire, la finalité du procès reste fondamentalement politique. Les préoccupations politiques sont donc toujours présentes et risquent d'interférer avec le respect des principes juridiques qui doivent normalement guider le travail de la justice.

Ainsi, le procès de Nuremberg, rappelle Guillaume Mouralis, «n'a cessé d'être travaillé par les tensions entre logiques pénales et diplomatiques »10. On le considérait volontiers comme une arène diplomatique traditionnelle où les magistrats nationaux étaient conscients de représenter leur pays. Or si la mission du diplomate est de défendre certains intérêts et de tenter de concilier des points de vue différents, il est demandé au juge d'être neutre et impartial et de prononcer des sanctions.

De fait, les préoccupations politiques et le souci des intérêts nationaux ont été constamment présents à Nuremberg. Ainsi, les magistrats soviétiques interdirent strictement d'évoquer le Pacte germano-soviétique et son protocole secret ainsi que le massacre de Katyn que leur propagande attribuait aux nazis. Mais les contraintes politiques jouèrent aussi, de façon plus subtile et plus indirecte, s'agissant de déterminer les contours du procès, notamment la définition des infractions et les limites de la compétence du tribunal. Une des préoccupations fondamentales des Américains était de ne pas créer un précédent qui justifierait à l'avenir une immixtion dans les affaires intérieures des États et donc une atteinte à leur souveraineté. Le chef du parquet américain, Robert Jackson, avait déclaré franchement, lors de la négociation du Traité, que « pour autant qu'il n'agresse pas ses voisins, un État reste libre de traiter ses habitants comme il l'entend ». S'explique ainsi la définition restrictive du crime contre l'humanité, arrimée à l'existence d'un plan concerté visant à déclencher une guerre d'agression, qui plaçait en dehors du champ de compétence du tribunal les persécutions des juifs allemands antérieures à l'entrée en guerre : se profilait ici la crainte des implications potentielles d'une définition trop large et générale des crimes raciaux commis par les nazis et de la perspective « de forger un outil juridique universel [...] susceptible d'ouvrir la voie à un examen international de l'ordre racial des États-Unis » 11. On peut ainsi,

7. FTP : Francs-tireurs et partisans ; FFI : Forces françaises de l'intérieur.

8. Plusieurs centaines de milliers de personnes sont arrêtées sans mandat entre août et octobre 1944. Alors que l'épuration officielle se soldera par 1536 peines de mort exécutées, l'épuration sauvage fait entre 8000 et 9000 morts (Alain BANCAUD, "L'épuration judiciaire à la Libération : entre légalité et exception », op. cit.) ; Denis SALAS, «La transition démocratique française après la Seconde Guerre mondiale », in ibid.

9. Alain BANCAUD, « La construction de l'appareil juridique », in Marc-Olivier BARUCH (dir.), Une poignée de misérables, Paris : Fayard, 2003

10. Le moment Nuremberg, p. 76

11. Ibid., p. 119-120. 
avec Guillaume Mouralis, analyser « la question raciale américaine [...] comme une contrainte $[\ldots]$ qui pesa lourdement sur le travail de catégorisation juridique des crimes à caractère raciste $»$.

D'une façon plus générale, les procès d'épuration ont tous été confrontés au même problème : comment atteindre l'objectif visé tout en respectant strictement le principe de nonrétroactivité de la loi pénale ? La conciliation étant de fait impossible, les juristes ont fait assaut d'imagination pour le contourner. Mais, en sens inverse, l'intangibilité du principe a parfois été instrumentalisée pour faire échec aux poursuites.

Une des difficultés majeures à laquelle se heurtait le jugement des criminels de guerre nazis à Nuremberg était leur incrimination sur la base d'infractions qui, au regard du droit pénal international en vigueur en 1940, soit n'existaient pas encore, soit ne pouvaient être imputées qu'aux États. Le tribunal a contourné l'obstacle en estimant que si l'accord de Londres n'avait pas de portée rétroactive, il pouvait néanmoins s'appliquer aux faits criminels commis antérieurement parce qu'ils étaient par nature incompatibles avec les lois et les coutumes de la guerre reconnues par la communauté internationale dès avant la Première Guerre mondiale 12 .

Hans Kelsen, dans un article publié en 1945 13, avait proposé une analyse plus subtile visant à relativiser la portée du principe de non-rétroactivité. S'agissant des crimes de guerre, écrivait-il, la question ne se pose pas car l'accord de Londres ne transforme pas un acte licite en acte illicite : il ajoute seulement la responsabilité individuelle des dirigeants allemands - une adjonction au demeurant prévisible puisqu'elle figurait dans le traité de Versailles pour la personne de Guillaume II. Les « atrocités » constitutives de crimes contre l'humanité peuvent être poursuivies sur la base du droit interne en vigueur au moment où elles ont été commises, comme le seraient des crimes ordinaires. S'agissant enfin des crimes contre la paix, même s'ils sont reconnus comme crimes internationaux depuis le pacte Briand-Kellogg, il est vrai qu'autoriser un tribunal international à infliger des peines de ce chef revient bien à édicter une règle rétroactive. Mais, ajoute-t-il, d'une façon qui paraît étonnante sous la plume d'un positiviste aussi convaincu, l'interdiction des lois rétroactives n'est pas un principe absolu, c'est un principe de justice qui entre en compétition avec d'autres principes de justice, en l'occurrence ici l'importance attachée à ce que les criminels de guerre soient poursuivis et punis : il peut donc légitimement être restreint pour tenir compte de cet impératif.

Le fait est qu'une conception stricte du principe de non-rétroactivité risque d'entraver l'entreprise d'épuration. Érigé en principe constitutionnel absolu par la Loi fondamentale de 1949, il a eu pour effet, sinon comme objet, de mettre un frein à la poursuite de la dénazification. La classe politique et les autorités judiciaires de la RFA ont en effet rejeté l'arsenal juridique mis en place par les Alliés avec les procès de Nuremberg et refusé d'appliquer rétroactivement les concepts de génocide et de crime contre l'humanité, forgés après la guerre et qui n'existaient pas d'un point de vue légal au moment des faits.

La Cour fédérale de justice, pour justifier malgré tout des condamnations - l'impunité totale aurait été une option politiquement difficile à tenir -, a dû recourir à des raisonnements subtils, fondés soit sur le droit positif, soit sur le droit naturel (nous y reviendrons plus loin) 14 .

12. Louis JoInet (dir.), Lutter contre l'impunité, Paris : La Découverte, coll. « Sur le vif», 2002, p. 105. L’auteur rappelle que le Pacte international sur les droits civils et politiques de 1966, en même temps qu'il proclame, dans son article 15, un principe de non-rétroactivité, précise qu'il n'empêche pas de condamner les auteurs d'actes qui, au moment où ils ont été commis, « étaient tenus pour criminels d'après les principes généraux du droit reconnus par l'ensemble des nations ».

13. « La règle interdisant les lois ex post facto et la poursuite des criminels de guerre de l'Axe »: voir Charles LEBEN, « Hans Kelsen et le châtiment des criminels de guerre de l'Allemagne nazie », Droit et Société, 102, 2019, p. 447.

14. Guillaume Mouralis, Une épuration allemande, op. cit., p. 296-297. 
Le même dilemme s'est posé à la Libération. La Résistance n'était pas unanime à vouloir respecter les principes juridiques traditionnels, à commencer par la non-rétroactivité des lois 15. L'épuration judiciaire porte la marque de débats et de compromis entre des conceptions contrastées. À un courant «radical» qui estimait que seule permettrait une véritable épuration une législation nouvelle dont il ne fallait pas hésiter à assumer la rétroactivité s'opposait un courant «légaliste» qui l'emporta au moins sur un point: pour éviter la rétroactivité, on utilisa en priorité les lois pénales en vigueur sous la III ${ }^{\mathrm{e}}$ République, notamment l'article 75 du Code pénal punissant la trahison. On créa toutefois un nouveau crime : celui d'indignité nationale, sanctionnée par la dégradation nationale, dont le caractère rétroactif n'était pas douteux mais qu'on justifia par le fait qu'il s'agissait en somme d'une loi plus douce.

De façon plus spécifique, s'agissant de l'épuration de la magistrature, les controverses autour du principe du secret du délibéré témoignent de la difficulté de concilier le strict respect des principes juridiques avec les objectifs assignés à l'épuration. La question s'avérait cruciale dès lors que la sévérité des peines prononcées par les juridictions d'exception, notamment la peine de mort, faisait partie des griefs justifiant l'exclusion de la magistrature, voire une condamnation pénale. Or le secret faisait obstacle à la recherche des responsabilités individuelles lorsque les décisions avaient été rendues collégialement 16.

Les instances d'épuration se sont donc trouvées placées devant une double alternative. Lever le secret, c'était contrevenir à un principe que les magistrats s'étaient engagés sous serment à respecter. Mais à vouloir l'observer strictement elles était confrontées à un dilemme : soit elles condamnaient solidairement les juges qui avaient participé à la sentence, alors même qu'elle n'avait pas nécessairement été votée à l'unanimité, substituant ainsi une responsabilité collective à la responsabilité individuelle, autre principe fondamental du droit pénal ; soit elles refusaient, précisément pour cette raison, de prononcer des condamnations, mais assuraient alors l'impunité à ceux que l'épuration avait justement pour but de sanctionner.

Toutes les instances concernées n'ont pas observé la même attitude. Dans le cadre de l'épuration disciplinaire, la commission chargée d'instruire les dossiers a accepté de lever le secret des délibérations à la marge : pour les seules juridictions d'exception et pour les quelques cas de condamnations à mort exécutées frappant des résistants officiellement reconnus. Le Conseil d'État, amené à contrôler les sanctions disciplinaires, a, quant à lui, opéré une distinction : s'agissant des sentences prononcées par les juridictions ordinaires, il a érigé la règle du secret en absolu, interdisant ainsi que soit attribuée à un magistrat la sévérité d'un jugement rendu (allant jusqu'à annuler la sanction prononcée contre un magistrat qu'on savait favorable à la collaboration et qui avait appliqué de façon particulièrement rigoureuse la législation raciale). Pour les juridictions d'exception, le Conseil d'État a esquivé la question du secret comme celle de la responsabilité : classant les sections spéciales et les tribunaux spéciaux dans la catégorie des juridictions «manifestement destinées à favoriser les entreprises de l'ennemi », il en a déduit que le simple fait d'avoir participé à leur fonctionnement était de nature à justifier une mesure d'épuration, dès lors que l'intéressé n'établit pas avoir siégé sous la contrainte

Devant les juridictions pénales, la défense a logiquement insisté sur le caractère fondamental et absolu du secret qui interdisait toute imputation individuelle à tel ou tel de la sentence rendue. Le ministère public, lui, a commencé par justifier la levée du secret par le fait que la procédure devant les juridictions d'exception étant viciée, la délibération était en tout état de cause entachée d'une nullité absolue... pour finalement conclure que la levée du

15. Alain BANCAUD, «L'épuration judiciaire à la Libération : entre légalité et exception », op. cit.

16. Alain BanCAUd et Denis SALAS, «Le jugement des juges des sections spéciales et le "principe sacré" du secret du délibéré », in Juger sous Vichy, juger Vichy. 
secret était de toutes façons inutile puisque la faute se situait en amont du vote et résidait dans le fait d'avoir accepté de siéger pour satisfaire les exigences des occupants.

\section{Quel droit appliquer ?}

\section{III.1. Le droit naturel contre le droit positif}

Il est clair que le droit positif, conjugué avec le principe de non-rétroactivité, était mal adapté pour traiter d'une criminalité hors normes. Cela explique la tentation d'invoquer le droit naturel pour s'affranchir des contraintes juridiques et la fréquente référence à la position exprimée par Gustav Radbruch en 1946, dans un article devenu célèbre 17. Si la loi positive doit être appliquée même si son contenu est injuste, écrivait-il, il n'en va plus de même lorsque la contradiction entre la loi positive et l'idée de justice atteint un degré tellement insupportable que cette loi doit céder la place à la justice. Car dans ce cas la loi positive n'est pas seulement un droit injuste, elle perd complètement son caractère juridique. Et de conclure que «mesurées à cet étalon, des parties entières du droit national-socialiste n'ont jamais obtenu le statut de droit juridiquement valable ».

Fritz Bauer, le procureur de Francfort à l'origine du procès d'Auschwitz 18, était de son côté persuadé que le droit en vigueur à l'époque des faits n'était pas un instrument adéquat pour juger les crimes nazis. Il estimait que seul un droit nouveau, donc rétroactif, pouvait permettre de juger les crimes sans précédent des nazis et militait pour le recours aux catégories forgées par les Alliés, ce à quoi, on l'a rappelé plus haut, les juristes allemands se sont refusés. Dans les procès de dénazification, la « formule» de Radbruch inspira toutefois les décisions de certains juges. Le tribunal constitutionnel avait par exemple affirmé, dans une décision rendue en 1953, concernant un crime commis sous le Troisième Reich, que dans des cas extrêmes il devait être possible de faire prévaloir le principe de justice substantielle (materielle Gerechtigkeit) sur celui de sécurité juridique (Rechtssicherheit) qui s'impose en général dans l'application de la loi positive 19. Mais les juridictions et les juristes allemands rejetèrent majoritairement les arguments jusnaturalistes. Sauf à absoudre l'ensemble des auteurs de crimes nazis, il fallait donc trouver un fondement aux poursuites dans le droit positif. Une forme de «positivisme réinterprétatif » 20 finit par s'imposer. On considéra que le Code pénal de 1871 resté dans ses grandes lignes inchangé, notamment son article 211 proscrivant le meurtre, pouvait servir de base juridique aux poursuites (ce qui revenait à traiter le génocide comme s'il s'agissait d'une criminalité « normale »). Une autre partie du droit en vigueur, sur lequel les accusés se fondaient pour justifier leurs actes, devait toutefois être considéré comme invalide : soit pour des raisons formelles car les ordres secrets de Hitler et certaines lois approuvées par le Reichstag n'avaient jamais été publiés au Journal officiel comme le prévoyait la constitution de Weimar (sic) jamais formellement abolie par les nazis ; soit en raison de leur contenu inconstitutionnel; soit enfin parce que les textes n'avaient pas été correctement interprétés à l'époque.

17. «Gesetzliches Unrecht und übergezetzliches Recht» (Injustice légale et droit supralégal), article présenté et traduit par Michael Walz, Archives de philosophie du droit, 39, 1994, p. 305-318.

18. Appelé aussi « procès de Francfort», ce procès où était mis en accusation le personnel du camp d'Auschwitz est le plus important de ceux intentés - tardivement ! - devant les tribunaux ouest-allemands contre des criminels nazis. Le nom de Fritz Bauer, le procureur de Francfort dont la persévérance a permis qu'il se tienne, y est étroitement associé. Voir Werner RENZ, "Le procès d'Auschwitz à Francfort (1963-1965). Préalables et déroulement », Revue d'histoire de la Shoah, 209, 2018, p. 591-613.

19. Cité par Guillaume Mouralis, Une épuration allemande, op. cit., p. 48.

20. Ibid., p. 297. 
Plus tard, lorsque vint le moment de l'épuration après la réunification allemande, la question se posa dans des termes analogues et la tentation du recours au droit naturel fut très forte comme le montrent les premiers procès des gardes-frontières jugés en 1991 comme auteurs de tirs mortels sur des fugitifs 21 . La thèse "positiviste » invoquée pour leur défense consistait à dire que, d'après les textes en vigueur, ils avaient le devoir d'empêcher une fuite, même au prix d'une vie humaine. La cour estima que ces textes n'avaient jamais eu la moindre validité et que les ordres qui en découlaient étaient nuls et non avenus, en se référant à la notion d' " injustice légale » ("gezetzliches Unrecht»), autrement dit aux thèses de G. Radbruch. Il existe, disait-elle, un noyau inaltérable du droit, qu'aucune loi ni aucun ordre ne saurait mettre en cause, auquel appartiennent les droits fondamentaux de la personne humaine tels que le droit à la vie. Pour étayer son raisonnement, le juge se référait à la jurisprudence du tribunal constitutionnel et notamment à la décision de 1953 citée plus haut. Et si ces arguments, disait le président du tribunal en 1991, avaient été forgés à la lumière des crimes nationaux-socialistes qui n'étaient pas comparables par leur nature et leur ampleur à ceux de la RDA, la protection de la vie humaine avait une portée universelle et ne pouvait dépendre du nombre de crimes commis. Dans ses conditions, la loi sur la frontière «ne méritait aucun respect et il fallait lui désobéir ». Les soldats et leurs supérieurs auraient dû et pu savoir en faisant appel à leur conscience que les tirs mortels à la frontière heurtaient les principes universellement reconnus du droit et de la justice.

Mais, lors d'un second procès, le raisonnement fut très différent, même s'il aboutit là encore à une condamnation : la juge affirmait que la liberté de quitter son pays ne relevait pas du «noyau inaltérable du droit» et que le conflit sécurité juridique vs justice substantielle était à résoudre au profit du premier principe. En RDA, la liberté d'émigration était déniée et les sanctions étaient dures et injustes, mais elles n'atteignaient pas l'insupportable degré d'injustice qui caractérisait la législation raciale nazie. Par conséquent, les crimes frontaliers ne relevaient pas des cas extrêmes évoqués par $\mathrm{G}$. Radbruch qui justifieraient une dérogation au principe de sécurité juridique. Ce qu'on pouvait en revanche reprocher aux gardes-frontières, c'était d'avoir perdu le sens des proportions, alors que la loi sur la frontière prévoyait expressément une réaction proportionnée. Le jugement fut confirmé en appel, mais sur un fondement intermédiaire entre la vision jusnaturaliste et la vision positiviste : pour les juges d'appel, la loi sur la frontière de 1982 devait être considérée comme invalide parce que contraire aux droits de l'homme universellement reconnus - des droits qui, depuis 1948, font incontestablement partie du droit international positif.

\section{III.2. La détermination des infractions punissables}

Nuremberg a constitué un grand moment d'innovation juridique : il s'agissait notamment de rendre juridiquement opératoire et de cristalliser en catégorie pénale effective le «crime contre l'humanité », cette notion indéterminée utilisée à de nombreuses reprises dans le passé et en dernier lieu pour caractériser les massacres des Arméniens 22. Guillaume Mouralis retrace minutieusement le cheminement par lequel on est finalement arrivé à la définition des infractions contenues dans l'accord de Londres, qui supposait de faire des choix entre des positions souvent incompatibles. Il évoque l'apport des " outsiders », auteurs des propositions les plus créatives et audacieuses mais qui ne seront finalement pas retenues, ou encore le rôle du représentant de l'Institute for Jewish Affairs, tentant en vain d'obtenir que soient inclus dans l'acte d'accusation les crimes commis par les puissances de l'Axe contre leurs propres nationaux et ceux ayant précédé l'entrée en guerre de l'Allemagne 23 . Il insiste enfin sur

21. Ibid., p. $42-54$.

22. Le moment Nuremberg, p. 108 et suiv.

23. Ibid., p. 87 et suiv. 
l'influence primordiale des «avocats d'élite» américains, qui sont aussi des avocats d'affaires, auxquels, dit-il, on doit les notions de «complot» («conspiracy») et d' « organisation criminelle », directement inspirées de leur pratique du droit du commerce 24.

On a évoqué plus haut les contraintes politiques qui ont pesé sur la catégorisation du crime contre l'humanité et notamment les efforts des Américains pour en cantonner la portée. Le jugement confirme sa subordination au contexte de guerre et elle se trouve réduite à la portion congrue. Entrée "par une très petite porte », la catégorie "s'est, du fait du jugement, volatilisée » 25 , le tribunal confirmant par ailleurs son incompétence pour reconnaître des persécutions raciales antérieures à l'entrée en guerre de l'Allemagne.

Le crime contre l'humanité a tenu encore moins de place dans les procès de dénazification : la loi $\mathrm{n}^{\circ} 10$ du Conseil de contrôle allié du 20 décembre 1945 qui servait de fondement aux poursuites diligentées devant les tribunaux allemands reprenait, dans son article 2, la liste des crimes punissables figurant dans le statut de Rome : crimes contre la paix, crimes de guerre, crimes contre l'humanité. Mais les juristes ouest-allemands, on l'a rappelé plus haut, ont refusé quasi unanimement de les utiliser du fait de leur caractère rétroactif, s'appuyant après 1949 sur la disposition de la Loi fondamentale interdisant les lois rétroactives, si bien que les principes de Nuremberg resteront inutilisés jusque dans les années 1990.

On trouve pourtant des références au crime contre l'humanité dans certaines décisions rendues par les tribunaux disciplinaires de la fonction publique sanctionnant les agents coupables d'avoir perpétré de tels crimes 26. Par ailleurs, les lois promulguées à partir de 1951, qui prévoient la réintégration des fonctionnaires ayant perdu leur poste dans le contexte de l'après-guerre - quelle qu'en soit la raison et y compris à la suite de la dénazification -, précisent que le bénéfice en est refusé à ceux «qui par leur comportement pendant la domination du national-socialisme ont enfreint les principes de l'humanité ou de l'État de droit $\gg$.

La même question du droit applicable s'est posée en France dès avant la Libération : châtier, certes, mais sur quelle base juridique, sous quelle forme et dans quelles limites 27 ? La doctrine de la France libre est fixée dès mars 1943 : il n’y a pas lieu d'inscrire dans la loi pénale française un nouveau crime de collaboration - donc de faire appel à des lois d'exception rétroactives - car celles de la République permettent de réprimer tous les actes englobés par ce terme. Il s'agit notamment de l'article 75 du Code pénal, dans sa version issue du décret-loi du 29 juillet 1939, qui punit de mort la trahison sous ses différentes formes. On crée malgré tout le crime d'indignité nationale sanctionné par la dégradation nationale. Et le jugement de l'ensemble de ces crimes, comme on le rappelle plus loin, sera remis à des juridictions spéciales.

\section{Qui est compétent, qui est légitime pour juger?}

Il faut d'abord faire le choix entre un tribunal national ou un tribunal international. À la conférence de Moscou, en octobre 1943, il avait été décidé que les criminels de guerre ayant agi à une échelle locale ou nationale seraient jugés sur le lieu de leurs crimes. Le sort des " grands criminels de guerre » ayant œuvré à l'échelle du Reich tout entier était renvoyé à une décision ultérieure - ce sera l'accord de Londres. Après l'expérience de Nuremberg, il a fallu attendre cinquante ans et la fin de la guerre froide, on le sait, pour que l'expérience soit

24. Ibid., p. 50-51.

25. La formule est du juge français, Henri Donnedieu de Vabres, cité par Guillaume Mouralis, ibid., p. 107.

26. Marie-Bénédicte VINCENT, « De la dénazification à la réintégration des fonctionnaires. Comment construire une éthique de la fonction publique en Allemagne de l'Ouest après 1945 ? », Vingtième Siècle. Revue d'histoire, 121, 2014, p. 3-19.

27. Jean-Louis CremieuX-BrilhaC, «Avant la Libération: la politique de la Justice à Alger et le procès Pucheu (19431944)», op. cit. 
renouvelée, d'abord avec la mise en place de juridictions ad hoc pour juger les crimes commis en ex-Yougoslavie et au Rwanda, puis avec la création de tribunaux hybrides ou mixtes, comme en Sierra Leone ou au Cambodge, pour finalement aboutir à la création d'une juridiction permanente avec la Cour pénale internationale.

Se pose aussi classiquement le choix entre des tribunaux ordinaires ou des tribunaux d'exception. Si, à la Libération, on a renoncé à instaurer une législation spécifique, hormis la création du crime d'indignité nationale, on a opté en revanche pour confier l'épuration à des juridictions ad hoc. L'ordonnance du 26 juin 1944 soustrait le jugement des crimes de trahison à leurs juges «naturels » que sont tribunaux militaires pour le remettre à des cours de justice spéciales, composées sur le modèle des cours d'assises. Pétain et Laval comparaîtront devant une Haute Cour de justice spécialement créée elle aussi. Enfin des chambres civiques seront instituées pour juger le crime d'indignité nationale. Et cette justice est loin de répondre aux critères d'une justice équitable 28 . La procédure est dérogatoire au droit commun, les droits de la défense sont imparfaitement respectés, les sentences sont rendues sans appel, et surtout elle n'obéit manifestement pas au critère d'impartialité - autant de dispositions qui entraînent une vive réaction de l'ordre des avocats. Le bâtonnier, Jacques Charpentier, va jusqu'à appeler à une "seconde résistance " contre ce qu'il considère comme un détournement de procédure qui discrédite la justice 29.

La partialité de la justice est de fait une critique récurrente adressée aux procès d'épuration, dénoncés, à l'instar du reste du procès de Nuremberg, comme une « justice des vainqueurs ». Si, à la Libération, la place des magistrats professionnels reste importante dans l'épuration judiciaire, puisqu'ils assument l'essentiel de l'instruction ainsi que la présidence et qu'ils participent au délibéré, on s'efforce autant que possible de nommer dans les cours de justice des magistrats résistants. Les jurés, eux, sont explicitement engagés : ceux des cours de justice et des chambres civiques sont sélectionnés sur une liste de citoyens n'ayant cessé de faire preuve de sentiments nationaux tout au long de l'Occupation. Les jurés de la Haute cour sont choisis parmi les parlementaires ayant voté contre les pleins pouvoirs en 1940, les personnalités appartenant aux mouvements de résistance, les prisonniers de guerre et les déportés politiques. En clair, on fait juger Vichy par ceux qui l'ont combattu 30.

Mais lorsqu'il s'agit de juger les criminels nazis, d'étranges scrupules se font jour sur la question de l'impartialité. Guillaume Mouralis rapporte ainsi que si l'on trouve au sein de la délégation américaine à Nuremberg un nombre important de juristes européens en exil et si les avocats juifs y sont surreprésentés par rapport à leur poids au sein du barreau américain 31 , les chefs du parquet américain et britannique refusèrent de donner des postes de responsabilité à des juristes juifs pour ne pas prêter le flanc au reproche d'une accusation partiale ou motivée par des considérations personnelles 32 .

Au procès d'Auschwitz, en 1963, le magistrat pressenti pour présider le tribunal devra se récuser sur la base de la "suspicion légitime ", au motif qu'il avait été lui-même persécuté pour raisons raciales et que des membres de sa famille étaient morts en déportation. Aux yeux du tribunal, la récusation se justifie car « du point de vue subjectif des accusés, on ne peut pas exclure qu'un juge faisant lui-même partie du cercle des personnes persécutées pour raisons raciales et ayant perdu des proches dans un camp de concentration ne soit pas entièrement libre et ne puisse leur faire face avec l'indépendance mentale requise » 33 . Fut également

28. Denis SALAS, «La transition démocratique française après la Seconde Guerre mondiale », op. cit.

29. Yves OzAnAm, « De Vichy à la résistance : le bâtonnier Jacques Charpentier », in Juger sous Vichy, juger Vichy.

30. Alain BANCAUD, «L'épuration judiciaire à la Libération : entre légalité et exception », op. cit.

31. Le moment Nuremberg, p. 26.

32. Ibid., p. 31.

33. Werner RENZ, « Le procès d'Auschwitz à Francfort (1963-1965). Préalables et déroulement », article cité. 
exempté de participation au procès, sur le même fondement, le fils du pasteur Martin Niemöller. Aucune objection ne fut en revanche formulée contre la nomination comme président d'un magistrat qui avait servi l'État sous le nazisme.

\section{L'appréciation des responsabilités : juger des individus ou des systèmes?}

\section{V.1. Nuremberg : la responsabilité individuelle au regard du droit international}

À Nuremberg s'est posé le problème de la mise en cause de la responsabilité individuelle des accusés alors que seule la responsabilité des États pouvait traditionnellement être recherchée pour violation du droit international. Toutefois, comme le relevait H. Kelsen en 1943 34, on avait déjà commencé à imaginer au moment du traité de Versailles une responsabilité individuelle de personnes coupables du déclenchement d'une guerre d'agression et prévu, s'il ne s'était pas réfugié aux Pays-Bas, de déférer Guillaume II, coupable d' " offense suprême contre la morale internationale» devant un tribunal composé de cinq juges nommés par chacune des cinq plus grandes puissances alliées. Les hésitations sont de courte durée puisque, dès 1943, à la troisième conférence de Moscou, il est clairement affirmé que la violation du droit international peut mettre en jeu non seulement la responsabilité collective des États mais aboutir au jugement des individus coupables d'actes criminels au regard du droit international.

\section{V.2. Le jugement d'Auschwitz : auteurs, complices ou auxiliaires?}

Au procès de Francfort, alors que le parquet disposait d'une liste d'environ 4000 membres de la SS d'Auschwitz, seuls furent poursuivis ceux contre lesquels des accusations concrètes avaient été portées : 60 personnes, dont 43 membres des SS et 15 détenus disposant de fonctions dans la hiérarchie concentrationnaire 35 . L'accusation considérait que toute personne ayant exercé une fonction à Auschwitz, parce qu'elle avait participé à la mise en œuvre d'un programme d'extermination, devait être mise en cause au moins comme complice 36 . Quelques décisions avaient été rendues en ce sens : ainsi, dans le procès du personnel du camp de Chelmno, en 1962, ou encore celui des camps d'extermination de Sobibór et Treblinka, les tribunaux avaient admis la responsabilité de toutes les personnes ayant servi dans ces camps de la mort qui avaient concouru de ce fait à un assassinat de masse. Mais le tribunal de Francfort considéra, conformément à la tendance dominante de la justice ouest-allemande en matière de crimes nazis, que seuls pouvaient être condamnés les donneurs d'ordres ou ceux dont on pouvait démontrer la participation directe aux crimes commis, ce qui équivalait à éluder la responsabilité des échelons intermédiaires. Et finalement, 14 personnes seulement furent condamnées à des peines de prison à perpétuité pour meurtre; les autres, considérées comme de simples « auxiliaires » n'ayant pas participé consciemment au crime et n'ayant fait qu'obéir aux ordres, se virent infliger des peines de prison légères ou furent acquittées.

Cette jurisprudence ne sera abandonnée que des années plus tard. Ainsi, Demjanjuk, ancien surveillant au camp d'extermination de Sobibór, est condamné en 2009 à cinq ans de prison pour « complicité de 27900 meurtres aggravés », le tribunal considérant que, même si aucune mort

34. Dans un texte intitulé «Responsabilité collective et responsabilité individuelle en droit international, concernant en particulier le châtiment des criminels de guerre » : voir Charles LEBEN, « Hans Kelsen et le châtiment des criminels de guerre de l'Allemagne nazie », article cité.

35. Voir Werner RENZ, « Le procès d'Auschwitz à Francfort (1963-1965). Préalables et déroulement », article cité.

36. Entretien avec Gerhard Wiese, un des membres de l'accusation, réalisé à l'occasion du cinquantième anniversaire du procès, <alencontre.org $\geq$, 15 janvier 2014. 
en particulier ne pouvait lui être imputée, l'accusé avait participé à la «machinerie de l'extermination ». Sera condamné de la même façon, en avril 2015, Oskar Gröning, ancien SS, en poste de 1942 à 1944 au camp d'extermination nazi d'Auschwitz-Birkenau, surnommé « le comptable d'Auschwitz» parce que, affecté à la «rampe » d'accès, il était chargé de récupérer les devises des déportés à leur arrivée. Bien que n'ayant jamais lui-même tué un détenu, il fut condamné pour " complicité de meurtres aggravée » dans l'extermination des 300000 juifs hongrois déportés entre mai et juillet 1944.

\section{V.3. Juger Vichy : les limites du "fait justificatif"}

Jusqu'à quel point l'obligation d'obéir aux lois de Vichy et aux ordres reçus pouvait-elle constituer un «fait justificatif» permettant d'échapper à une condamnation ? Reconnaître le fait justificatif de façon trop large risquait d'aboutir à une " déculpabilisation collective"; mais exclure entièrement l'existence d'une telle contrainte serait revenu à transformer l'épuration en mouvement d'élimination sans limite, dangereux au moment où la reconstruction de l'État était une priorité 37.

Les hésitations à cet égard sont patentes. En témoigne la rédaction des ordonnances qui encadrent l'épuration. L'ordonnance du 6 décembre 1943 impose de faire la distinction « entre les hommes qui se sont bornés à exécuter des ordres sans avoir l'autorité nécessaire pour les discuter et ceux qui, allant au-delà de leurs strictes obligations professionnelles, se sont sciemment associés à une politique antinationale ou ont manifestement dépassé dans la répression l'exercice normal de leurs fonctions ». L'ordonnance du 26 juin 1944 écarte les poursuites contre les fonctionnaires qui se sont bornés à la stricte exécution des ordres reçus, exclusive de toute initiative personnelle, ou à l'unique accomplissement d'obligations professionnelles sans participation volontaire à un acte antinational. Elle exclut toutefois une telle excuse dans des cas particulièrement graves (dénonciation, actes individuels de violence, renseignements à l'ennemi...). Elle prévoit enfin que les lois, ordres et autorisations de Vichy ne constituent pas le «fait justificatif» prévu par le Code pénal lorsque le prévenu avait la faculté de se soustraire à leur exécution.

Dans le cas spécifique de la magistrature, les critères de la compromission étaient particulièrement difficiles à dégager 38 . Comme le rappelait une circulaire rédigée en février 1944 par un groupe de magistrats résistants du tribunal de la Seine, le personnel judiciaire est par nature placé en dehors de la sphère d'activité politique, il est obligé par définition d'appliquer des lois qui s'imposent à lui du moment qu'elles émanent de l'autorité apparemment légale.

Si le caractère punissable de certains actes comme les réquisitions de peines de mort, les propos public favorables à la collaboration, l'excès de zèle dans la répression, n'était pas contesté, la question de la participation aux juridictions d'exception faisait l'objet d'appréciations divergentes 39. À l'encontre de ceux qui souhaitaient éliminer l'ensemble des magistrats ayant siégé dans les cours spéciales et les tribunaux d'État, la circulaire nuance : « Si le magistrat a rempli ses fonctions avec un esprit partisan, il a manqué à son devoir, s'il n'a fait que s'incliner sans aucun zèle devant la loi dont il s'est efforcé de tempérer l'injustice, aucune sanction immédiate ne saurait être prise contre lui. » Dans la pratique, la simple participation aux juridictions d'exception ne fut pas jugée suffisante pour justifier à soi seule une sanction 40 : ne furent sanctionnés par la commission centrale d'épuration de la

37. Alain BANCAUD, «L'épuration judiciaire à la Libération : entre légalité et exception », op. cit.

38. Pierre Truche et Denis SAlaS, « René Linais, juge à la section spéciale », in Juger sous Vichy, juger Vichy.

39. Jean-Paul JEAN, «Quel regard porter sur les magistrats ayant siégé dans les juridictions d'exception sous l'Occupation? ».

40. Alain BANCAUD, «L'épuration des épurateurs », p. 197 et suiv. 
magistrature que ceux qui avaient manifesté une trop grande sévérité, et notamment requis ou prononcé des peines de mort. Le Conseil d'État, saisi de recours contre les sanctions disciplinaires, adopta toutefois sur ce point une position plus radicale : à ses yeux, le fait d'avoir accepté de siéger, sans y être contraint, dans une juridiction d'exception quelle qu'elle soit suffisait à fonder une mesure d'épuration. Lorsqu'ils ont été déférés devant les juridictions pénales, les magistrats ont été condamnés pour leur appartenance à des groupes collaborationnistes ou nazis, mais rarement sur la base de leur activité judiciaire.

\section{V.4. La RDA : les rouages d'un système?}

La question des critères de jugement pour apprécier les responsabilités s'est reposée inéluctablement dans les procès d'épuration de la RDA. Plusieurs figures des mouvements civiques est-allemands estimaient du reste que la perspective individuelle de la justice pénale était inadaptée pour mettre au jour les mécanismes impersonnels de la criminalité d'État 41 . L'hésitation des juges transparaît par exemple dans le procès mettant en cause le ministre de la Défense et deux autres personnalités éminentes du régime : ils étaient accusés d'être les coauteurs de 68 meurtres commis entre 1961 et 1989 du fait qu'ils avaient joué un rôle clé dans la mise en place du régime frontalier. Mais le tribunal, tout en les déclarant coupables, prend ses distances par rapport à l'acte d'accusation : il relève qu' '« ils étaient eux-mêmes les maillons d'un système » et en déduit que "leur comportement doit être évalué à l'aune du contexte historique global » marqué par la guerre froide. Dans ce cas, le fait de n'avoir été que les rouages d'un système a donc joué en faveur des accusés pour atténuer leur responsabilité 42 .

\section{Quelles leçons pour l'avenir ?}

Les juristes et les hommes politiques américains avaient bien conscience, on l'a rappelé plus haut, des retombées potentielles d'une mise en cause internationale des persécutions raciales qui déboucherait sur la critique des lois en vigueur aux États-Unis. Dès l'arrivée de Hitler au pouvoir, les intellectuels afro-américains et les black lawyers avaient pointé la parenté entre les lois de Nuremberg et la législation raciale américaine et établi un parallèle entre les persécutions antijuives et la pratique du lynchage du Klan 43. Et le Pittsburgh Courier du 29 septembre 1945 commentait l'accord de Londres en ces termes : "Quelle ironie! Les États-Unis signent un accord en vertu duquel les "persécutions raciales" constituent un crime contre la société internationale. » Les avocats de la défense, au cours du procès, n'avaient du reste pas manqué d'insister sur l'inspiration que les théoriciens du racisme nazi avaient pu trouver dans les lois Jim Crow 44.

Le procès de Nuremberg a-t-il pu dès lors servir de ressource dans les mobilisations d'aprèsguerre aux États-Unis contre le racisme? Les avocats des droits civiques, nous dit Guillaume Mouralis, ont rarement invoqué directement les nouveaux principes du droit pénal international, tels que le crime contre l'humanité, dans les procès destinés à contester le racisme sous ses différentes formes, qu'il s'agisse des lois explicitement discriminatoires ou des violences tolérées ou encouragées par les autorités. Il est vrai que la définition du crime

41. Guillaume MouraLIS, Une épuration allemande, op. cit., p. 146.

42. Ibid., p. 63-69.

43. Le moment Nuremberg, p. 125 et 143.

44. On désignait ainsi les textes qui, dans les États du sud, organisaient la ségrégation raciale. La plupart sont restés en vigueur jusqu'à l'adoption du Civil Rights Act en 1964. 
contre l'humanité était verrouillée puisque arrimée au contexte de guerre, taillée sur mesure pour les criminels nazis

En revanche, les acquis de Nuremberg furent fréquemment invoqués en dehors des tribunaux par les militants associatifs. Le mot "génocide», en particulier, figure dans les pétitions adressées aux Nations unies par les organisations afro-américaines à partir de 1947 45. En 1951 est rendu public un document intitulé : "We charge genocide » qui réclame réparation pour les crimes commis par le gouvernement des États-Unis contre le peuple noir, sur la base d'un acte d'accusation dressé sur le modèle de Nuremberg.

Plus tard, la forme du procès international a été utilisée comme arme militante pour dénoncer les crimes commis par les États-Unis au Vietnam : le tribunal Russel s'était donné pour mission, en 1966, d'enquêter sur les crimes de guerre américains et d'en «juger» les auteurs sur la base des principes de Nuremberg et du droit humanitaire issu des conventions de Genève. Le modèle a été largement repris par la suite, et c'est dans son sillage qu'a été créé en en 1979 par Lelio Basso le Tribunal permanent des peuples 46. Le succès des tribunaux d'opinion a incontestablement à voir avec le peu de confiance placé dans les institutions officielles, malgré la mise en place des juridictions pénales internationales, pour lutter contre l'impunité.

On peut se demander, en conclusion, si le modèle judiciaire de l'épuration qui a prévalu après la guerre n'est pas devenu obsolète, même s'il a été réactivé après la chute du régime de la RDA. Il paraît aujourd'hui largement concurrencé voire remplacé par celui de la «justice transitionnelle » dont les objectifs dépassent de loin le châtiment des coupables, incluant le droit à la vérité, la reconnaissance des victimes et leur droit à la réparation et enfin la réconciliation nationale. Reste à savoir si ces objectifs sont véritablement compatibles entre eux : le travail des commissions "vérité-justice-réconciliation » a montré qu'il risquait de fragiliser la dimension pénale, au détriment de la lutte contre l'impunité 47.

Faute de place ont été laissées de côté une série de questions qui auraient mérité tout autant l'attention. On ne peut donc qu'inciter les lecteurs à se reporter aux ouvrages et aux textes commentés pour y retrouver ce qui n'a pu être pris en compte dans cet $\grave{A}$ propos.

45. Ibid., p. 168 et suiv.

46. $<$ https://intercoll.net/Tribunal-Permanent-des-Peuples>.

47. Voir Louis JoINET (dir.), Lutter contre l'impunité, op. cit., notamment p. 64 et suiv. 